\title{
Simulation of Submicron Silicon Diodes with a Non-Parabolic Hydrodynamical Model Based on the Maximum Entropy Principle
}

\author{
O. MUSCATO ${ }^{\mathrm{a}, *}$ and V. ROMANO ${ }^{\mathrm{b}, \dagger}$ \\ ${ }^{a}$ Dipartimento di Matematica e Informatica, Universitá di Catania, Viale Andrea Doria 6 - 95125 Catania, Italy; \\ ${ }^{\mathrm{b}}$ Dipartimento Interuniversitario di Matematica, Politecnico di Bari Via E. Orabona 4-70125 Bari, Italy
}

\begin{abstract}
A hydrodynamical model for electron transport in silicon semiconductors, free of any fitting parameters, has been formulated in $[1,2]$ on the basis of the maximum entropy principle, by considering the energy band described by the Kane dispersion relation and by including electron-non polar optical phonon and electron-acoustic phonon scattering.

In [3] the validity of this model has been checked in the bulk case. Here the consistence is investigated by comparing with Monte Carlo data the results of the simulation of a submicron $n^{+}-n-n^{+}$silicon diode for different length of the channel, bias voltage and doping profile.

The results show that the model is sufficiently accurate for CAD purposes.
\end{abstract}

Keywords: Charge transport; Hydrodynamical model; Electron devices

\section{THE MATHEMATICAL MODEL}

Modeling modern submicron electron devices requires an accurate description of charge transport in order to cope with high-field phenomena that cannot be described satisfactorily within the framework of the drift-diffusion equations (which do not comprise energy as a dynamical variable and also are valid only in the quasi-stationary limit). Generalizations of the drift-diffusion equations have been sought which would incorporate energy as a dynamical variable and also would not be restricted to quasi-stationary situations. These models are loosely speaking called hydrodynamical models, comprising also the so-called energy models. Hydrodynamical models are obtained from the infinite hierarchy of the moment equations of the Boltzmann transport equation by a suitable truncation procedure. Most implemented hydrodynamical models suffer from serious theoretical drawbacks due to the ad hoc treatment of the closure problem [4]. In this paper we present a recently introduced moment approach in which the closure procedure is based on the maximum

*Corresponding author. Tel.: + 39 (095) 738-3033, Fax: + 39 (095) 733-7007, e-mail: muscato@dmi.unict.it
$\dagger$ †e-mail: romano@dmi.unict.it 
entropy principle and the energy bands are described by the Kane dispersion relation [1,2].

Let us consider the semiclassical Boltzmann transport equation for electrons in semiconductors

$$
\frac{\partial f}{\partial t}+v^{i}(\mathbf{k}) \frac{\partial f}{\partial x^{i}}-\frac{e E^{i}}{\hbar} \frac{\partial f}{\partial k^{i}}=\mathcal{C}[f],
$$

where $f(\mathbf{x}, t, \mathbf{k})$ is the one particle distribution function giving the probability of finding an electron, in the position $\mathbf{x}$ and at time $t$, with a state belonging to a small volume of the first Brillouin zone $\mathcal{B}$ centered at the state of wave vector k. $\mathcal{C}[f]$ describes the effects due to scattering with phonons, impurities and with other electrons. E represents the self-consistent electric field which is related to the electron distribution function through Poisson's equation

$$
\mathbf{E}=-\nabla_{x} \phi, \quad \varepsilon \Delta \phi=-e\left(N_{D}-N_{A}-n\right)
$$

where $\phi$ is the electric potential, $e$ the elementary charge, $N_{D}$ and $N_{A}$ the donor and acceptor densities respectively, $\varepsilon$ the dielectric constant and $n$ the particle density. We shall consider the unipolar case and therefore the hole concentration will be not included. The electron group velocity $\mathbf{v}$ is given by $\mathbf{v}=(1 / \hbar) \nabla_{\mathbf{k}} \mathcal{E}$, where $\mathcal{E}(\mathbf{k})$ is the energy of the considered conduction band measured from the band minimum.

In the approximation of the Kane dispersion relation, $\mathcal{E}$ is expressed as

$$
\mathcal{E}(k)[1+\alpha \mathcal{E}(k)]=\frac{\hbar^{2} k^{2}}{2 m^{*}}, \quad \mathbf{k} \in \mathcal{R}^{3}
$$

where $\alpha$ is the non parabolicity parameter, $m^{*}$ the effective electron mass (which is $0.32 \mathrm{~m}_{e}$ in $\mathrm{Si}), \hbar \mathbf{k}$ the crystal momentum, which is assumed to vary in all the space $\mathcal{R}^{3}$, and $\hbar$ the Planck constant $h$ divided by $2 \pi$. Concerning the collision term, we take into account the most relevant interactions for silicon, that is those between electrons and non-polar optical phonons and between electrons and acoustic phonons (the values of these coupling constants can be found in [4]). The electron-electron scattering and scattering of electrons with ionized impurities, imperfections and vacancies will not be considered.

The macroscopic balance equations are deduced by taking the moments of the Boltzmann transport equations, that is by multiplying Eq. (1) by a weight function $\psi(\mathbf{k})$ and integrating over $\mathcal{B}$. If we consider the set of weight functions $1, \hbar \mathbf{k}, \mathcal{E}$ and $\mathcal{E} \mathbf{V}$ and assume as equivalent the six $\mathrm{X}$-valleys, one gets the following macroscopic balance equations

$$
\begin{gathered}
\frac{\partial n}{\partial t}+\frac{\partial\left(n V^{i}\right)}{\partial x^{i}}=0 \\
\frac{\partial\left(n P^{i}\right)}{\partial t}+\frac{\partial\left(n U^{i j}\right)}{\partial x^{j}}+n e E^{i}=n C_{P}^{i} \\
\frac{\partial(n W)}{\partial t}+\frac{\partial\left(n S^{j}\right)}{\partial x^{j}}+n e V_{k} E^{k}=n C_{W} \\
\frac{\partial\left(n S^{i}\right)}{\partial t}+\frac{\partial\left(n F^{i j}\right)}{\partial x^{j}}+n e E_{j} G^{i j}=n C_{W}^{i}
\end{gathered}
$$

where

$$
\begin{aligned}
& n=\int_{\mathcal{R}^{3}} f d^{3} \mathbf{k} \text { is the electron density, } \\
& V^{i}=\frac{1}{n} \int_{\mathcal{R}^{3}} f v^{i} d^{3} \mathbf{k} \text { is the } \text { average electron velocity, } \\
& W=\frac{1}{n} \int_{\mathcal{R}^{3}} \mathcal{E}(k) f d^{3} \mathbf{k} \text { is the }
\end{aligned}
$$

average electron energy,

$S^{i}=\frac{1}{n} \int_{\mathcal{R}^{3}} f v^{i} \mathcal{E}(k) d^{3} \mathbf{k}$ is the energy flux,

$P^{i}=\frac{1}{n} \int_{\mathcal{R}^{3}} f \hbar k^{i} d^{3} \mathbf{k}=m^{*}\left(V^{i}+2 \alpha S^{i}\right)$ is the

average crystal momentum,

$U^{i j}=\frac{1}{n} \int_{\mathcal{R}^{3}} f v^{i} \hbar k^{j} d^{3} \mathbf{k}$ is the

flux of crystal momentum,

$G^{i j}=\frac{1}{n} \int_{\mathcal{R}^{3}} \frac{1}{\hbar} f \frac{\partial}{\partial k_{j}}\left(\mathcal{E} v_{i}\right) d^{3} \mathbf{k}$, 


$$
\begin{aligned}
F^{i j}= & \frac{1}{n} \int_{\mathcal{R}^{3}} f v^{i} v^{j} \mathcal{E}(k) d^{3} \mathbf{k} \text { is the } \\
& \text { flux of energy flux, } \\
C_{P}^{i}= & \frac{1}{n} \int_{\mathcal{R}^{3}} \mathcal{C}[f] \hbar k^{i} d^{3} \mathbf{k} \text { is the production of } \\
& \text { the crystal momentum balance equation, } \\
C_{W}= & \frac{1}{n} \int_{\mathcal{R}^{3}} \mathcal{C}[f] \mathcal{E}(k) d^{3} \mathbf{k} \text { is the production of } \\
\text { the energy balance equation, } & \frac{1}{n} \int_{\mathcal{R}^{3}} \mathcal{C}[f] v^{i} \mathcal{E}(k) d^{3} \mathbf{k} \text { is the production } \\
& \text { of the energy flux balance equation. }
\end{aligned}
$$

The previous moment equations do not constitute a set of closed relations because of the fluxes and production terms. Therefore constitutive assumptions must be prescribed.

If we assume as fundamental variables $n, V^{i}, W$ and $S^{i}$, which have a direct physical interpretation, the closure problem consists in expressing $P^{i}, U^{i j}$, $F^{i j}$ and $G^{i j}$ and the moments of the collision term $C_{P}^{i}, C_{W}$ and $C_{W}^{i}$ as functions of $n, V^{i}, W$ and $S^{i}$.

The Maximum Entropy Principle (hereafter MEP) leads to a systematic way for obtaining constitutive relations on the basis of information theory. According to the MEP if some moments $M_{A}$ are known, the distribution function $f_{M E}$ which can be used to evaluate the unknown moments of $f$, corresponds to the extremal of the entropy functional under the constraints that it yields exactly the known moments $M_{A}$, that is

$$
\int_{\mathcal{R}^{3}} \psi_{A} f_{M E} d^{3} \mathbf{k}=M_{A}
$$

with $\psi A(\mathbf{k})$ weight function entering into the definition of the assigned moments $M_{A}$.

Since the electrons interact with the phonons describing the thermal vibrations of the ions placed at the points of the crystal lattice, in principle we should deal with a two component system (electrons and phonons). However, if one considers the phonon gas as a thermal bath at constant temperature $T_{L}$, only the electron component of the entropy must be maximized.
Moreover, since the electron gas is in the most cases sufficiently dilute, one can use the expression of the entropy obtained as limiting case of that arising in the Fermi statistics

$$
s=-k_{B} \int_{\mathcal{R}^{3}}(f \log f-f) d^{3} \mathbf{k} .
$$

If we introduce the lagrangian multipliers $\Lambda A$, the problem to maximize $s$ under the constraints (9) is equivalent to maximize $s^{\prime}=\Lambda^{A} M_{A}-s$, the Legendre transform of $s$, without constraints, $\delta s^{\prime}=0$. If $n, V^{i}, W$ and $S^{i}$ are assumed as fundamental variables, then the vector of the weight functions and the vector of the respective lagrangian multipliers, are

$$
\begin{aligned}
\left(\psi^{A}\right) & =(1, \mathbf{v}, \mathcal{E}, \mathcal{E} \mathbf{v}) \text { and } \\
\left(\Lambda_{A}\right) & =\left(\lambda, k_{B} \lambda_{i}, k_{B} \lambda^{W}, k_{B} \lambda_{i}^{W}\right)
\end{aligned}
$$

with $\lambda$ lagrangian multiplier relative to the density, $\lambda^{W}$ lagrangian multiplier relative to the energy, $\lambda_{i}$ lagrangian multiplier relative to the velocity and $\lambda_{i}^{W}$ lagrangian multiplier relative to the energy flux. Therefore the maximum entropy distribution function reads

$$
f_{M E}=\exp \left[-\left(\frac{1}{k_{B}} \lambda+\lambda^{W} \mathcal{E}+\lambda_{i} v^{i}+\lambda_{i}^{W} v^{i} \mathcal{E}\right)\right]
$$

with $\Lambda A$ functions of the moments $M_{A}$.

In order to get the dependence of the $\Lambda A$ 's from the $M_{A}$, one has to invert the constraints (9). Monte Carlo simulations for electron transport in Si show that the anisotropy of $f$ is small [4] even far from equilibrium. Upon such a consideration we make the ansatz of small anisotropy for $f_{M E}$. Formally we introduce a small anisotropy parameter $\delta$, assume that the multipliers are analytic in $\delta$ and expand them around $\delta=0$. Once the expression of the lagrangian multipliers has been obtained, by evaluating the constraints (8) with $f=f_{M E}$, one gets the closure relations. Explicit constitutive equations have been obtained in [1] for high order fluxes and in [2] for the production 
terms up to second order in $\delta$. However it has been found in [5] that the first order model is sufficiently accurate for the applications and avoids some irregularities due to the nonlinearities as in the parabolic band case [6]. Here we do not report the explicit form of the closure relations: the interested reader can see $[1,2,5]$.

\section{NUMERICAL RESULTS}

We test the hydrodynamical model by simulating a $n^{+}-n-n^{+}$silicon diode, that models the channel of a MOSFET. The $n^{+}$regions are $0.1 \mu \mathrm{m}$ long while the channel has different lengths, several doping profiles have been considered and all the
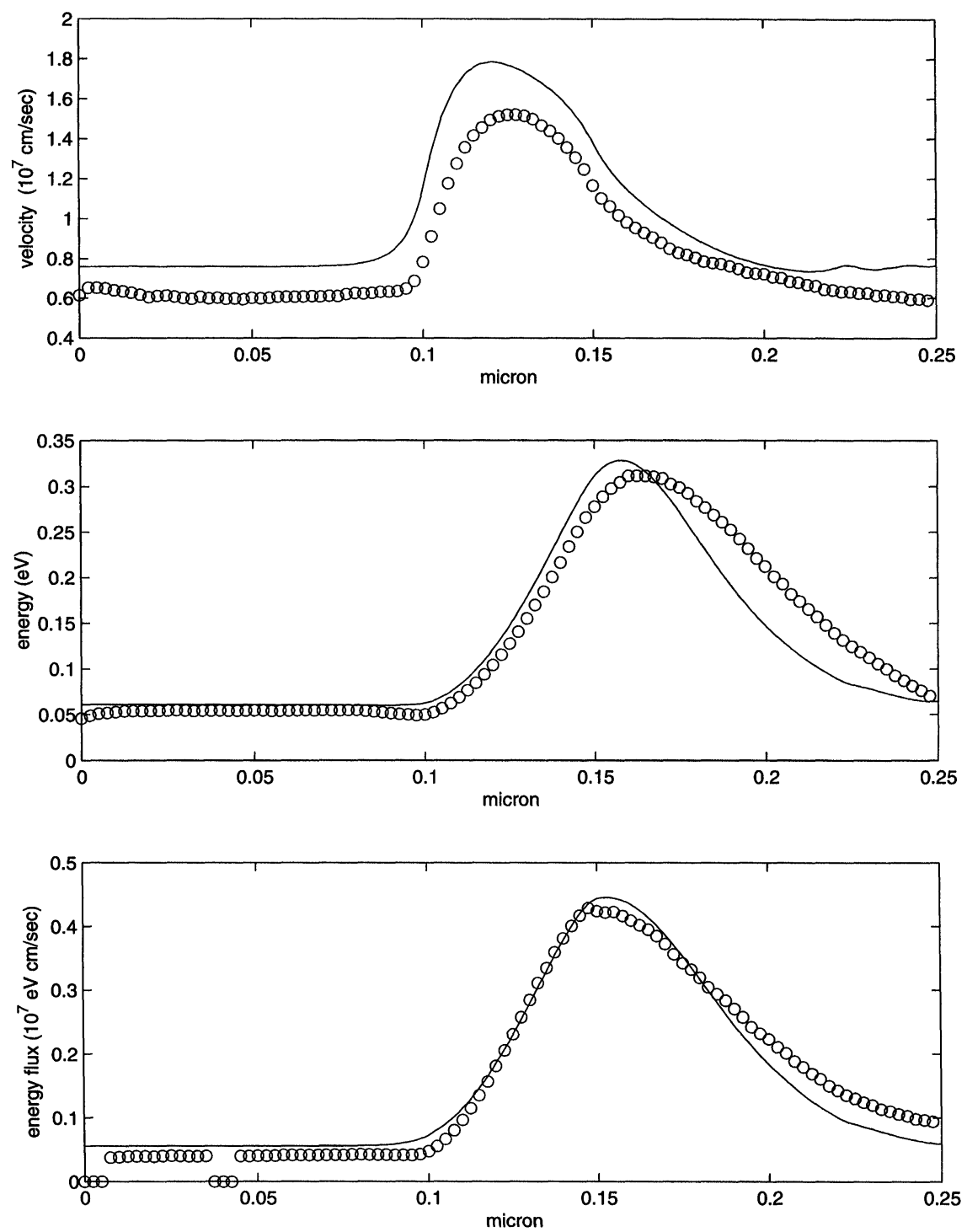

FIGURE 1 Profile of velocity, energy and energy flux obtained by the hydrodynamical model (continuous line) and by Monte Carlo simulation (circles). On the left column it is shown the case of channel 0.4 micron, $V_{b}=2$ Volts and doping density in the $n^{+}$ region $5 \times 10^{17} \mathrm{~cm}^{-3}$ and in the $n$ region $0.02 \times 10^{17} \mathrm{~cm}^{-3}$. On the right column it is reported the case of channel 0.3 micron, $V_{b}=$ 1 Volt and doping density in the $n^{+}$region $10^{18} \mathrm{~cm}^{-3}$ and in the $n$ region $\times 10^{16} \mathrm{~cm}^{-3}$. 

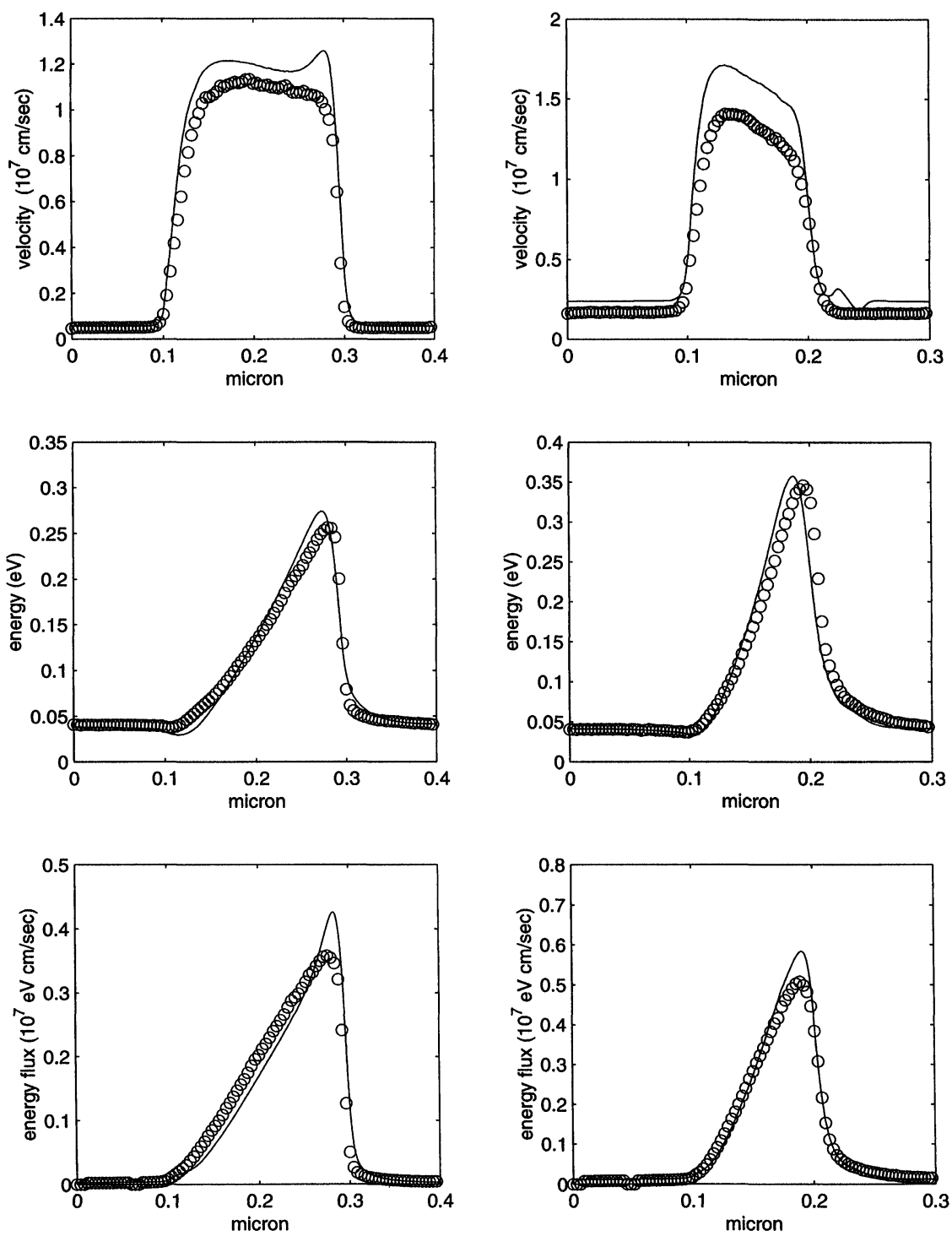

FIGURE 2 Profile of velocity, energy and energy flux obtained by the hydrodynamical model (continuous line) and by Monte Carlo simulation (circles). On the left column it is shown the case of channel 0.2 micron, while on the right column it is reported the case of channel 0.1 micron. In both cases $V_{b}=1$ Volt and doping density in the $n^{+}$region $10^{18} \mathrm{~cm}^{-3}$ and in the $n$ region $\times 10^{16} \mathrm{~cm}-3$.

simulations are performed at room temperature, $T_{L}=300 \mathrm{~K}$.

Since the evolution equations form a quasilinear hyperbolic system of Partial Differential Equations in physical region of the dependent variables characterized by $n>0$ and $W>0$ [5], for the numerical integration we have used the method followed in [6]. The doping profile is represented by a stepwise function and a grid with 400 nodes has been used. The stationary solution is reached within a few picoseconds (about five), after a short transient with wide oscillations. In this paper we compare this solution with the Monte Carlo data obtained by the simulator Damocles [7]: we underline that our hydrodynamical model is free on any fitting 

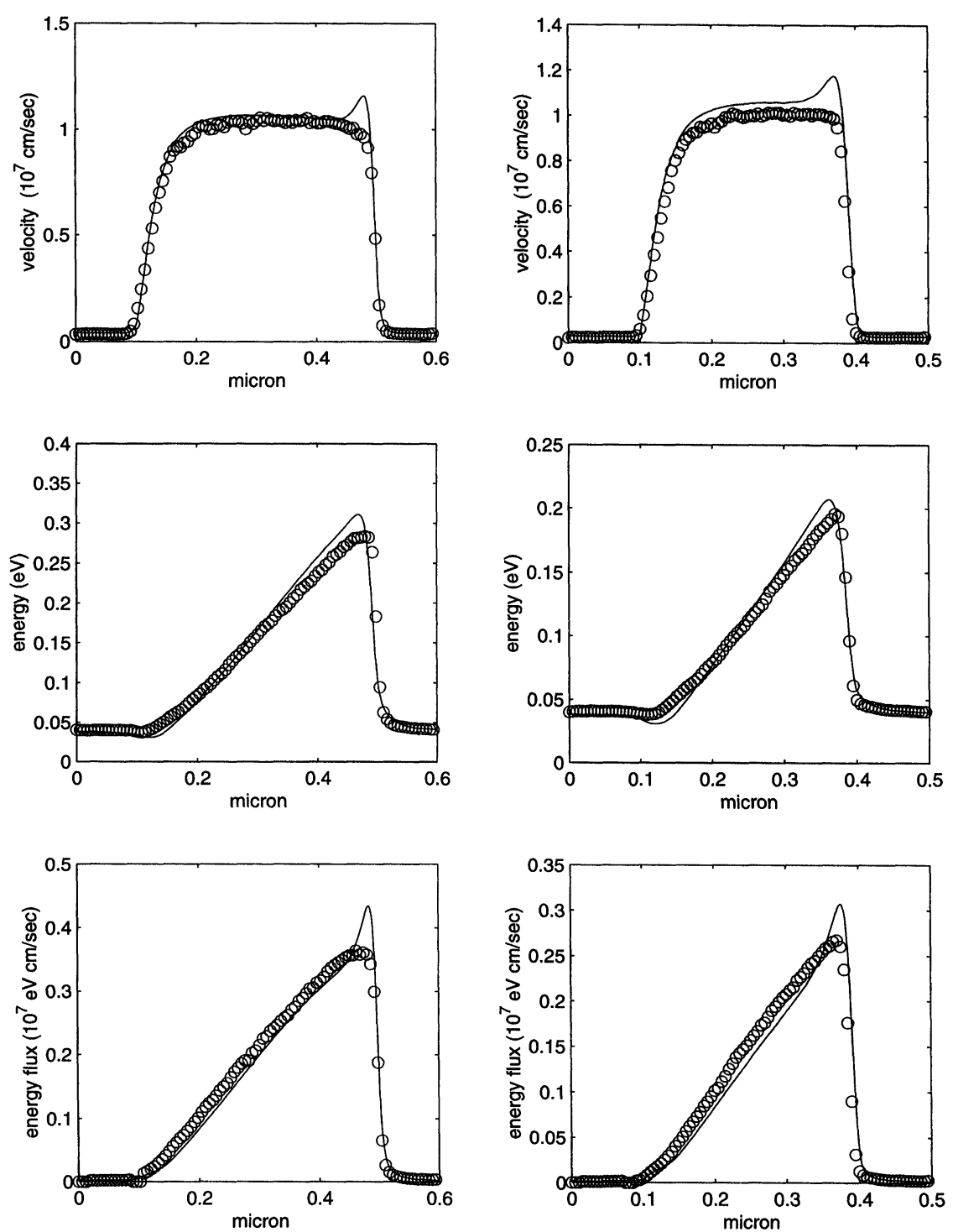

FIGURE 3 Profile of velocity, energy and energy flux obtained by the hydrodynamical model (continuous line) and by Monte Carlo simulation (circles) in the case of channel 0.05 micron, $V_{b}=1$ Volt and doping density in the $n^{+}$region $5 \times 10^{17} \mathrm{~cm}^{-3}$ and in the $n$ region $0.02 \times 10^{17} \mathrm{~cm}^{-3}$.

parameters, because the same coupling constants which are in Damocles have been used. Moreover in the Monte Carlo simulations we have retained only the same scattering mechanisms as those considered in the hydrodynamical model (e.g., electron-electron interaction is not included).

As first test we consider the case of the channel of length $0.4 \mu \mathrm{m}$ and bias voltage $V_{b}=2$ Volts. In
Figure 1 (left column) the stationary solution (after $5 \mathrm{ps}$ ) is plotted. In the same figure (right column) we report also the results for the channel of length $0.3 \mu \mathrm{m}$, with $V_{b}=1$ Volt and a different doping profile. In both simulation the error for all the field variables with respect to the Monte Carlo solution is less that $10 \%$ unless in the second junction where a small spurious spike in the velocity is present. 
In the Figure 2 we report the results for channels of length 0.2 and $0.1 \mu \mathrm{m}$ with $V_{b}=1$ Volt. The mean difference with Monte Carlo data is again about $10 \%$ for energy and energy-flux. For the electron velocity one has in the case of $0.2 \mu \mathrm{m}$ channel the same percent error that becomes about $20 \%$ for the $0.1 \mu \mathrm{m}$ channel.

As last test we show the results of a channel of length $0.05 \mu \mathrm{m}$ and $V_{b}=1$. Again the agreement is rather satisfactory for energy and energy-flux while the error in the velocity is about $20 \%$. Probabily in this last case in order to achieve a better agreement with MC data, additional moment equations must be taken into account.

The shown results indicate that the hydrodynamical model based on the maximum entropy principle is very promising as CAD tool. It gives very satisfactory results for submicron $n^{+}-n-n^{+}$silicon diode between 0.2 and $0.4 \mu \mathrm{m}$ while for shorter devices the results are less accurate but still acceptable and very challenging with respect to other existing hydrodynamical models. Improvements for dealing more accurately with nano-scale devices are under consideration.

\section{Acknowledgments}

This work has been partially supported by MURST, Fondi $60 \%$ and project Problems in kinetic theory, by CNR project Modelli matematici per semiconduttori. Progetto speciale: metodi matematici in fluidodinamica e dinamica molecolare, grant number 96.03855.CT01 and by TMR program Asymptotic methods in kinetic theory, grant number ERBFMRXCT970157.

\section{References}

[1] Anile, A. M. and Romano, V. (1999). "Non parabolic band transport in semiconductors: closure of the moment equations", Cont. Mech. Thermodyn., 11, 307.

[2] Romano, V. (2000). "Non parabolic band transport in semiconductors: closure of the production terms in the moment equations", Cont. Mech. Thermodyn., 12,31 .

[3] Anile, A. M., Muscato, O. and Romano, V., "Moment equations with maximum entropy closure for carrier transport in semiconductors devices: validation in bulk silicon", to appear in VLSI Design [preprint archive TMR project on Asymptotic methods in kinetic theory, http:// www.math.tuberlin.de/ $\sim \mathrm{tmr}]$.

[4] Muscato, O., "Monte Carlo valuation of the transport coefficients in a $n^{+}-n-n^{+}$silicon diode", to appear in COMPEL [preprint archive TMR project on Asymptotic methods in kinetic theory, http://www.math.tu-berlin.de / $\sim \mathrm{tmr}]$

[5] Romano, V., "Nonparabolic band hydrodynamical model of silicon semiconductors and simulation of electron devices" [preprint archive TMR project on Asymptotic methods in kinetic theory, http://www.math.tu-berlin. $\mathrm{de} / \sim \mathrm{tmr}]$.

[6] Anile, A. M., Romano, V. and Russo, G., "Extended hydrodynamical model of carrier transport in semiconductors", to appear in SIAM J. Appl. Math. [preprint archive TMR project on Asymptotic methods in kinetic theory, http://www.math.tu-berlin.de/ $\sim \mathrm{tmr}]$.

[7] Laux, S. E., Fischetti, M. V. and Frank, D. J. (1990). IBM J. Res. Develop., 34. 

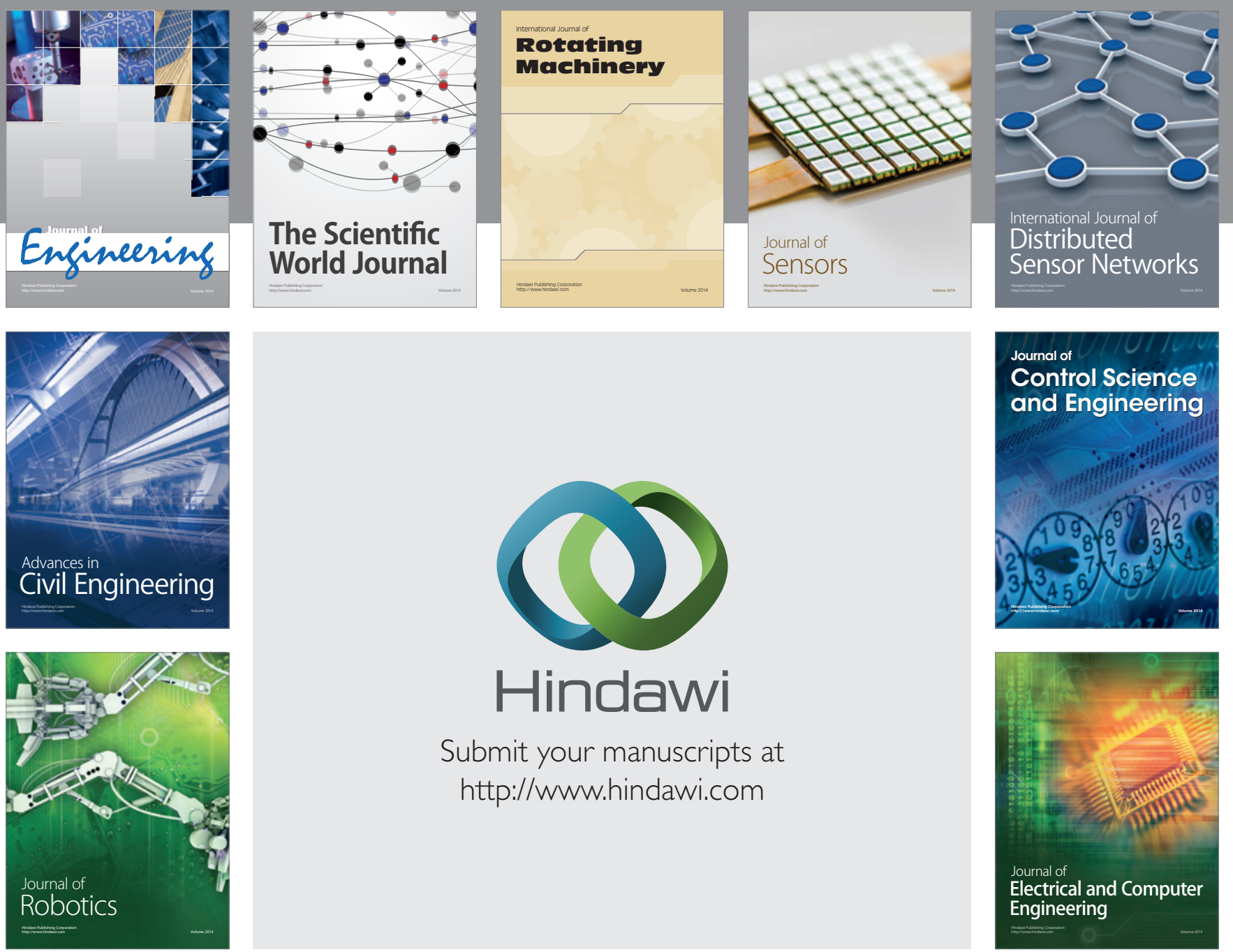

Submit your manuscripts at

http://www.hindawi.com
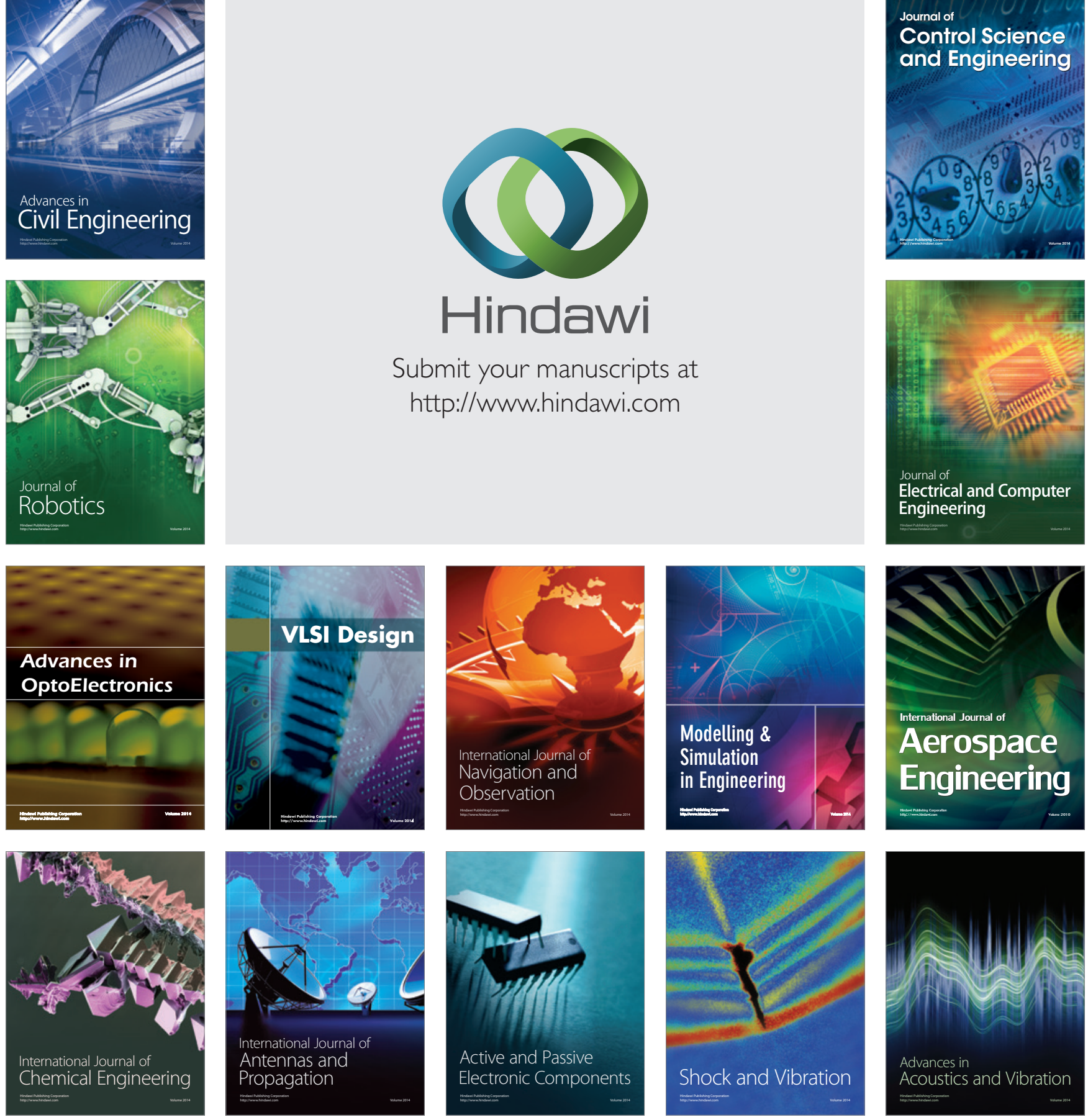